\section{ORIGINAL RESEARCH}

\author{
H.R. Underhill \\ C. Yuan \\ V.L. Yarnykh \\ B. Chu \\ M. Oikawa \\ L. Dong \\ N.L. Polissar \\ G.A. Garden \\ S.C. Cramer \\ T.S. Hatsukami
}

\title{
Predictors of Surface Disruption with MR Imaging in Asymptomatic Carotid Artery Stenosis
}

BACKGROUND AND PURPOSE: Surface disruption, either ulceration or fibrous cap rupture, has been identified as a key feature of the unstable atherosclerotic plaque. In this prospective observational study, we sought to determine the characteristics of the carotid lesion that predict the development of new surface disruption.

MATERIALS AND METHODS: One hundred eight asymptomatic individuals with $50 \%-79 \%$ carotid stenosis underwent carotid MR imaging at baseline and at 3 years. Multicontrast imaging criteria were used to determine the presence or absence of calcification, LRNC, intraplaque hemorrhage, and surface disruption. Volume measurements of plaque morphology and the LRNC and calcification, when present, were collected.

RESULTS: At baseline, 21.3\% (23/108) of participants were identified with a surface disruption. After 3 years, $9(10.6 \%)$ of the remaining 85 individuals without disruption at baseline developed a new surface disruption during follow-up. Among all baseline variables associated with new surface disruption during regression analysis, the proportion of wall volume occupied by the LRNC (percentage LRNC volume; OR per $5 \%$ increase, $2.6 ; 95 \% \mathrm{Cl}, 1.5-4.6$ ) was the strongest classifier (AUC $=0.95$ ) during $\mathrm{ROC}$ analysis. New surface disruption was associated with a significant increase in percentage LRNC volume $(1.7 \pm 2.0 \%$ per year, $P=.035)$

CONCLUSIONS: This prospective investigation of asymptomatic individuals with $50 \%-79 \%$ stenosis provides compelling evidence that LRNC size may govern the risk of future surface disruption. Identification of carotid plaques in danger of developing new surface disruption may prove clinically valuable for preventing the transition from stable to unstable atherosclerotic disease.

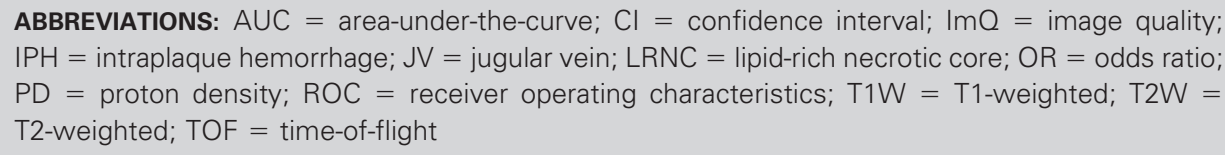

S urface disruption, defined as the presence of ulceration or fibrous cap rupture, is a key element of the unstable atherosclerotic lesion. The occurrence of microemboli during presurgical monitoring with transcranial Doppler has been correlated with ulceration on histology after carotid endarterectomy. ${ }^{1}$ Park et $\mathrm{al}^{2}$ reported that $77 \%$ of carotid lesions removed from symptomatic patients had an ulceration, which was significantly higher than the prevalence of ulceration in plaques removed from asymptomatic patients with highgrade stenosis. Similarly, fibrous cap rupture has been shown to occur more frequently in symptomatic plaques compared with lesions from asymptomatic arteries within ${ }^{3}$ and among $^{4}$ patients. Moreover, prospective data have demonstrated that surface conditions at baseline were predictive of future cerebrovascular events. ${ }^{5}$ While a strong association between sur-

Received May 28, 2009; accepted after revision July 14.

From the Departments of Radiology (H.R.U., C.Y., V.L.Y., B.C., M.O., L.D.), Neurology (G.A.G.), and Surgery (T.S.H.), University of Washington, Seattle, Washington; MountainWhisper-Light Statistical Consulting (N.L.P.), Seattle, Washington; and Department of Neurology (S.C.C.), University of California, Irvine, California.

This work was supported by grants from the National Institutes of Health (R01-HL073401, R01 HL 67406, P01-HL072262, and T32-HL07838).

Please address correspondence to Thomas S. Hatsukami, MD, Vascular Imaging Lab, University of Washington, 815 Mercer St, Box 358050, Seattle, WA 98109; e-mail: tomhat@u.washington.edu

Indicates open access to non-subscribers at www.ajnr.org

DOI 10.3174/ajnr.A1842 face disruption and symptomatic neurologic events has become apparent, features that predispose a lesion to the development of surface disruption remain ambiguous. Identification of plaque attributes that are present before the development of surface disruption may afford the opportunity to escalate medical intervention and reduce the risk of developing an unstable lesion.

Carotid MR imaging has enabled the in vivo assessment of both the morphologic ${ }^{6}$ and compositional characteristics of the carotid artery wall. Via histologic validation, multisequence carotid MR imaging has been proved as able to identify surface disruption and to detect and quantify the LRNC, calcification, and IPH. ${ }^{7-10}$ Subsequently, multisequence carotid MR imaging has been used for associating plaque features with neurologic events, ${ }^{3-5,11}$ following the natural history of carotid atherosclerosis, ${ }^{12,13}$ and for monitoring the response to therapy. ${ }^{14-16}$

In this study, we sought to determine carotid plaque characteristics that predict the development of a new surface disruption. Accordingly, we designed a prospective study that used carotid MR imaging to evaluate the morphology and features of carotid atherosclerotic disease at baseline and at 3-year follow-up.

\section{Materials and Methods}

\section{Study Sample}

Individuals with at least 1 carotid artery with $50 \%-79 \%$ stenosis as determined by duplex sonography by using Strandness criteria ${ }^{17}$ were 
serially recruited from the diagnostic vascular sonography laboratory at the University of Washington Medical Center and the Veterans Affairs Puget Sound Health Care System. The artery with 50\%-79\% stenosis was designated as the index artery and was selected for serial imaging by carotid MR imaging. In the case where the right and left carotid arteries had 50\%-79\% stenosis, the index artery was randomly assigned. Subjects were asymptomatic with respect to their carotid disease on the index side before enrollment. The study sample described herein represents the subset of individuals previously reported by Takaya et $\mathrm{al}^{5}$ who underwent a follow-up carotid MR imaging 3 years after their baseline scan.

At both scanning sessions, participants provided answers to a standardized health questionnaire. At the baseline scanning, subjects had their height, weight, and mean systolic blood pressure from both arms recorded. After the initial MR imaging evaluation, all participants were given a telephone interview every 3 months during the period of observation to assess the development of stroke, transient ischemic attack, or amaurosis fugax consistent with the side of the index artery. Participants who gave a history of a neurologic event on the telephone interview were scheduled for an interval visit and underwent a detailed neurologic examination by the study neurologist and review of hospital records.

The study procedures and consent forms were reviewed and approved by the institutional review board before study initiation. Exclusion criteria were prior carotid endarterectomy on the index carotid artery, prior radiation therapy to the neck, age $>80$ years at the time of enrollment, or contraindication for MR imaging. Medications were not a criterion for exclusion, and all patients were managed medically by their primary care providers for the duration of the observation period.

\section{MR Imaging Protocol}

From July 22, 1999, to January 18, 2007, 120 individuals initially asymptomatic were serially evaluated with carotid MR imaging on a 1.5T scanner (Signa Horizon EchoSpeed, Version 5.8; GE Healthcare, Milwaukee, Wisconsin) by using bilateral 4-element, phased array surface coils (Pathway MRI, Seattle, Washington). A standardized multicontrast protocol $^{18}$ for carotid MR imaging was used to obtain 2D T1-weighted PD-weighted, and T2-weighted black-blood images and 3D TOF bright-blood angiography. All images were obtained with an FOV of $16 \mathrm{~cm}$, matrix size of $256 \times 256$, and 2 signal-intensity averages. In-plane acquisition resolution was $0.62 \times 0.62 \mathrm{~mm}$. Twelve axial images were acquired with a $2-\mathrm{mm}$ section thickness, for a total longitudinal coverage of $24 \mathrm{~mm}$, centered at the bifurcation of the index artery. After acquisition, all images underwent zero-filled interpolation to an image size of $512 \times 512$. Total acquisition time for all images was approximately 30 minutes. Of note, the hardware, pulse sequences, and imaging protocol were identical for all subjects during the 3 years between baseline and follow-up scanning.

\section{Image Review}

Two reviewers, blinded to the time point and clinical information, matched the axial images of the index artery between time points on the basis of the distance from the bifurcation of the carotid artery. All matched axial locations for the index artery were evaluated for ImQ (4-point scale: 1 = poor, $4=$ excellent) and were interpreted by reviewers reaching a consensus opinion. For images with $\operatorname{ImQ} \geq 2$ at both time points, image analysis software (CASCADE, ${ }^{19}$ Seattle, Washington) was used to draw the lumen and outer wall boundaries. Lumen volume, wall volume, and total vessel volume (lumen volume

\begin{tabular}{|c|c|c|}
\hline & $\begin{array}{l}\text { Mean } \pm \text { SD } \\
\text { or } \%\end{array}$ & Range \\
\hline Age (yrs) & $70.7 \pm 9.3$ & $44-88$ \\
\hline Male sex & 89.0 & \\
\hline Body mass index & $27.9 \pm 4.9$ & $18.9-54.3$ \\
\hline Systolic blood pressure $(\mathrm{mm} \mathrm{Hg})^{\text {a }}$ & $144.9 \pm 21.0$ & $98.5-194.0$ \\
\hline \multicolumn{3}{|l|}{ Smoking } \\
\hline Never smoked & 13.0 & \\
\hline Quit & 51.4 & \\
\hline Active & 35.5 & \\
\hline Diabetes mellitus & 26.0 & \\
\hline History of coronary artery disease & 50.0 & \\
\hline History of hypertension & 88.0 & \\
\hline History of hypercholesterolemia & 86.1 & \\
\hline Statin therapy & 72.0 & \\
\hline Minimum lumen area $\left(\mathrm{mm}^{2}\right)$ & $17.8 \pm 10.0$ & $3.1-51.4$ \\
\hline Percentage wall volume & $39.8 \pm 13.1$ & $18.7-82.9$ \\
\hline Presence of LRNC & 48.1 & \\
\hline Presence of calcification & 70.4 & \\
\hline Presence of IPH & 29.6 & \\
\hline Percentage LRNC volume ( $n=52)$ & $16.5 \pm 15.9$ & $0.3-62.2$ \\
\hline $\begin{array}{l}\text { Percentage calcification volume } \\
\qquad(n=76)\end{array}$ & $37.0 \pm 21.8$ & $3.1-82.3$ \\
\hline
\end{tabular}

a Missing data for $n=2$.

+ wall volume) were used to calculate percentage wall volume (100\% $\times$ wall volume / total vessel volume), a measure of plaque burden similar to that proposed by Nissen et $\mathrm{al}^{20}$ during imaging investigations of the coronary arteries. Multicontrast imaging criteria previously validated with histology were used to determine the presence or absence of calcification, LRNC, IPH, and surface disruption (ulceration or fibrous cap rupture). ${ }^{7-10}$ Volume measurements of the LRNC and calcification, when present, were collected. The proportion of each component (LRNC and calcification) relative to the wall volume was also determined (eg, percentage LRNC volume $=$ LRNC volume / wall volume $\times 100 \%$ ). Reproducibility for each morphology and compositional metric have been previously reported (inter-reader, ${ }^{8,9,21,22}$ interscan $\left.^{14,21,23,24}\right)$. IPH was treated as a dichotomous variable (present versus absent) due to decreased reproducibility of volume measurements compared with the other metrics. ${ }^{8}$

\section{Data Analysis}

Minimum lumen area was determined by the minimum value across all matched locations for each artery. Summary statistics for each metric are presented as mean \pm SD for all arteries included in each analysis. The independent $t$ test was used to compare differences in continuous metrics of plaque morphology and composition at baseline between lesions with and without a surface disruption. The Fisher exact test was used to compare differences in the distribution of dichotomous variables (eg, presence versus absence of IPH) between lesions with and without a surface disruption. Univariate binary logistic regression analysis by using all of the clinical and baseline arterial variables identified in Table 1 was conducted to identify statistically significant predictors of a new surface disruption. An OR and its 95\% CI are reported for statistically significant associations. Meaningful multivariate analysis could not be performed due to the small number of new surface disruptions that occurred during the study period. ROC and AUC were used to assess the accuracy of classification for each statistically significant metric from univariate analysis. AUC and its associated 95\% CI are reported. Annualized change in continuous metrics was compared with zero by using the 1-sample $t$ 

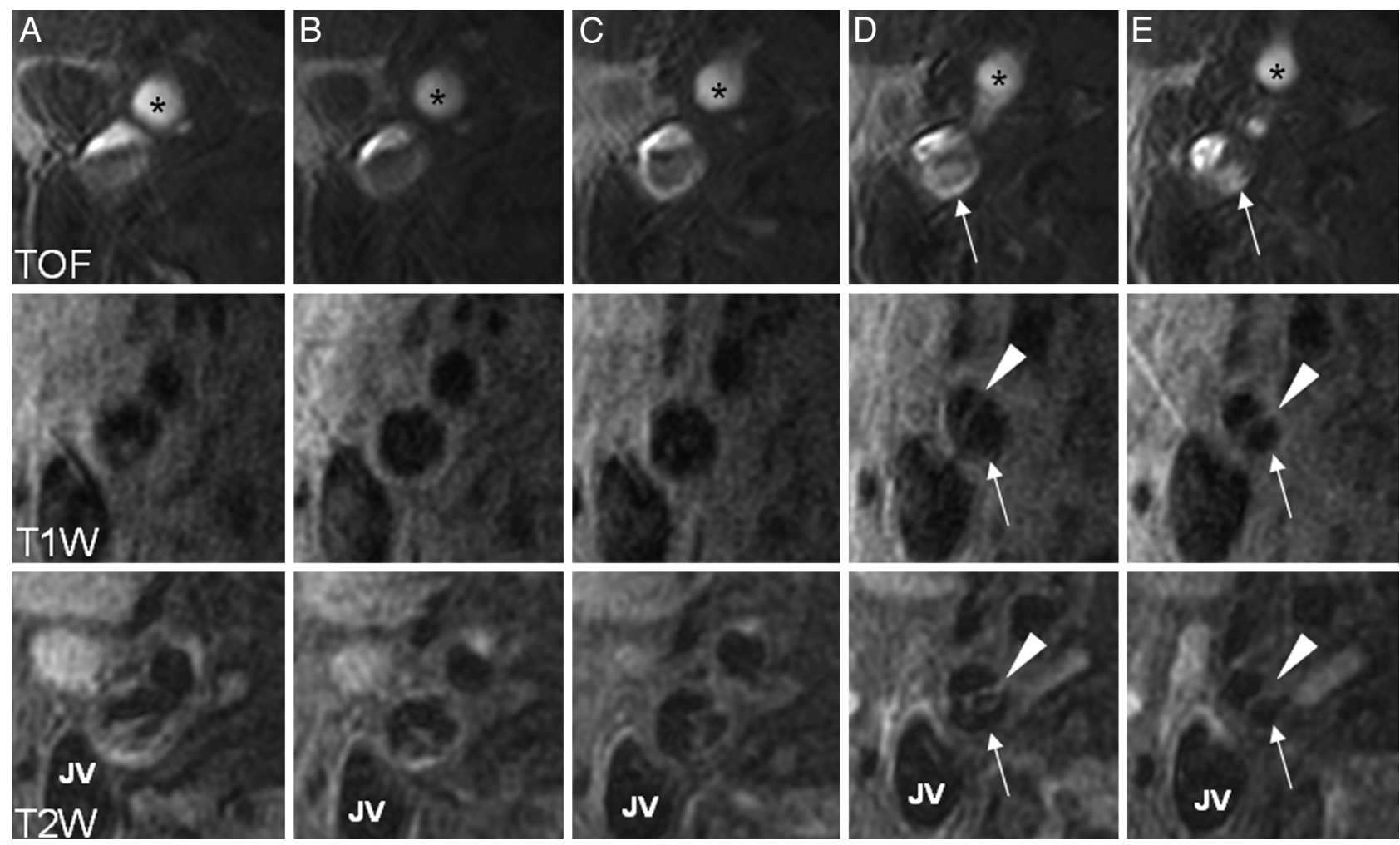

Fig 1. Multicontrast images from the baseline examination of a 68-year-old man. The axial images are consecutive sections of the right internal carotid artery beginning at the flow divider (column A). There are substantial flow artifacts due to the proximity of the bifurcation. In column D, 2 separate lumens are developing in the internal carotid artery as indicated by 2 levels of intensity on TOF and a band of tissue (arrowheads) separating the true lumen from a penetrating ulcer (arrow). The ulceration extends into the adjacent more distal section (column E). There is no evidence of an LRNC in this artery. Asterisk indicates the lumen of the external carotid artery.

Table 2: Comparison $(n=85)$ of baseline features that develop a new surface disruption at follow-up versus lesions whose surface remains intact

\begin{tabular}{|c|c|c|c|c|c|}
\hline & \multicolumn{2}{|c|}{ Univariate Regression } & \multicolumn{3}{|c|}{ Independent $t$ Test or Fisher Exact Test } \\
\hline & OR (95\% CI) & $\operatorname{AUC}(95 \% \mathrm{CI})$ & $\begin{array}{l}\text { Present } \\
(n=9)\end{array}$ & $\begin{array}{c}\text { Absent } \\
(n=76)\end{array}$ & $\begin{array}{c}P \\
\text { Value }\end{array}$ \\
\hline Minimal lumen area $\left(\mathrm{mm}^{2}\right)$ & 3.7 per $5-\mathrm{mm}^{2}$ decrease $(1.6-8.7)$ & $0.87(0.77-0.97)$ & $8.9 \pm 4.5$ & $20.3 \pm 10.5$ & $<.001$ \\
\hline Percentage wall volume & 2.6 per $5 \%$ increase $(1.5-4.6)$ & $0.85(0.71-0.98)$ & $62.5 \pm 8.9$ & $50.6 \pm 7.2$ & $<.001$ \\
\hline Percentage LRNC volume & 2.6 per $5 \%$ increase (1.5-4.4) & $0.95(0.90-1.00)$ & $16.2 \pm 11.3$ & $1.6 \pm 4.4$ & .005 \\
\hline Prevalence of LRNC $(\%)^{a}$ & & $0.86(0.77-0.94)$ & 100 & 28.9 & $<.001$ \\
\hline Prevalence of IPH (\%) & 12.3 for presence of IPH (2.7-56.8) & $0.73(0.53-0.94)$ & 55.6 & 9.2 & .55 \\
\hline
\end{tabular}

a OR $(95 \% \mathrm{Cl})$ could not be calculated because $100 \%$ of lesions with a new surface disruption had an LRNC at baseline.

test. The independent $t$ test was used to compare differences in annualized change between lesions that developed a surface disruption and lesions without a surface disruption. All calculations were made by using SPSS 12.0 for Windows (SPSS, Chicago, Illinois). Statistical significance was defined as a value of $P<.05$.

\section{Results}

The mean interval between the baseline and follow-up scanning was $3.0 \pm 0.2$ years. One hundred eight $(90.0 \%)$ subjects demonstrated image quality $\geq 2$ at both time points. The demographics and baseline arterial characteristics for the evaluable population $(N=108)$ are presented in Table 1 . The mean longitudinal coverage of the artery that matched between time points was $17.6 \pm 3.2 \mathrm{~mm}$.

\section{Surface Disruption at Baseline}

At baseline, 21.3\% (23/108) of individuals were identified with a surface disruption. While not all lesions with a surface dis- ruption exhibited an LRNC (Fig 1), compared with the group without surface disruption, a significantly higher prevalence of LRNC (91.3\% versus $36.5 \%$, respectively; $P<.001$ ) and IPH (87.0\% versus $14.1 \%$, respectively; $P=.035$ ) was found. The group with surface disruption compared with the group without had a smaller minimal lumen area (13.1 \pm 4.9 versus $19.1 \pm 10.7 \mathrm{~mm}^{2}$, respectively; $\left.P<.001\right)$, a larger percentage wall volume $(60.6 \pm 8.8$ versus $51.8 \pm 8.2 \%$, respectively; $P<$ $.001)$, a larger percentage LRNC volume $(16.1 \pm 14.9$ versus $3.1 \pm 7.1 \%$, respectively; $P<.001)$, and a smaller percentage calcification volume $(1.5 \pm 2.4$ versus $5.4 \pm 6.0 \%$, respectively; $P<.001)$.

\section{Baseline Plaque Features and New Surface Disruption during Follow-up}

Of the 85 individuals without surface disruption at baseline, $10.6 \%(9 / 85)$ developed a surface disruption at 3 years (Fig 2). Metrics from Table 1 with a statistically significant association 

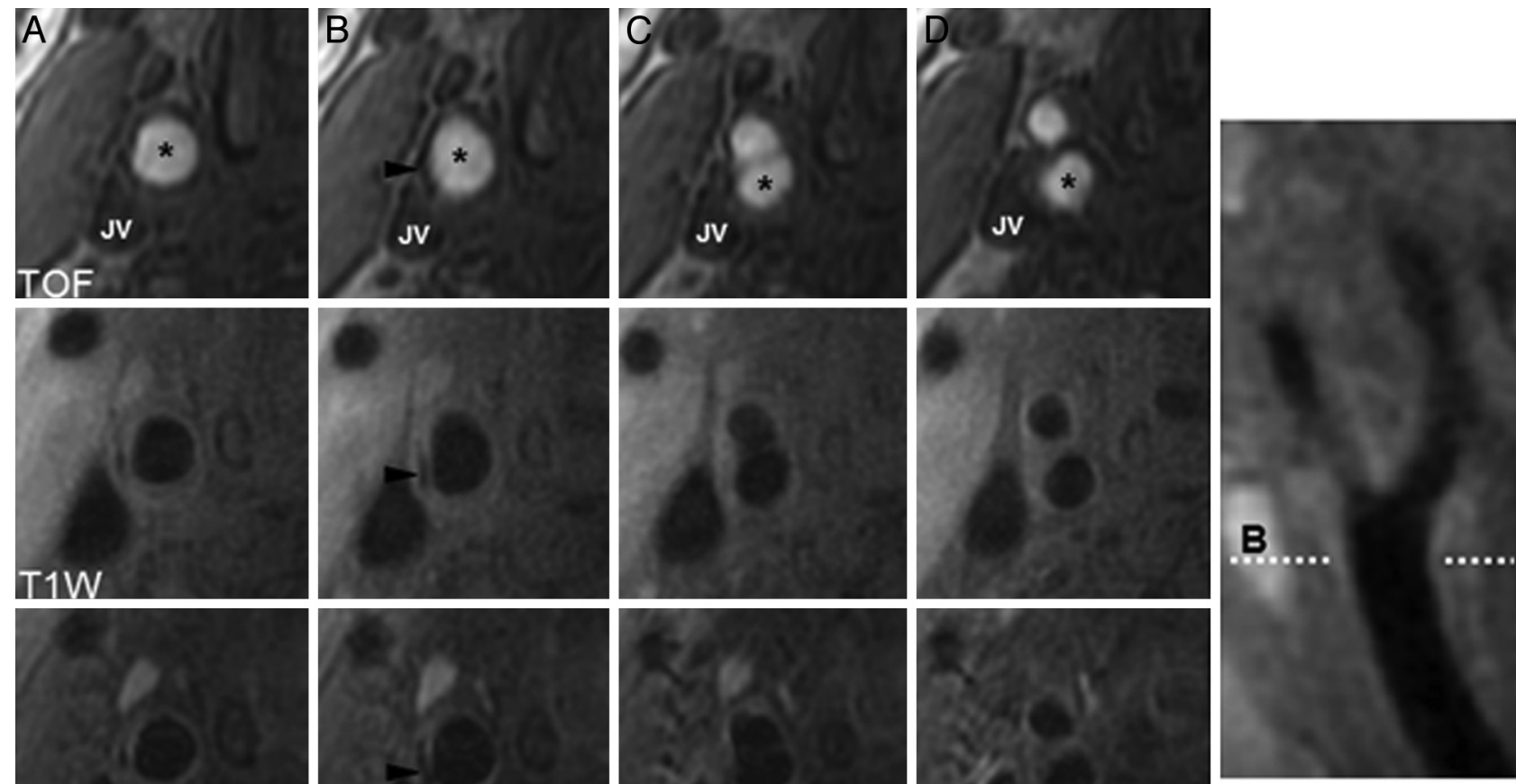

\section{T2W}
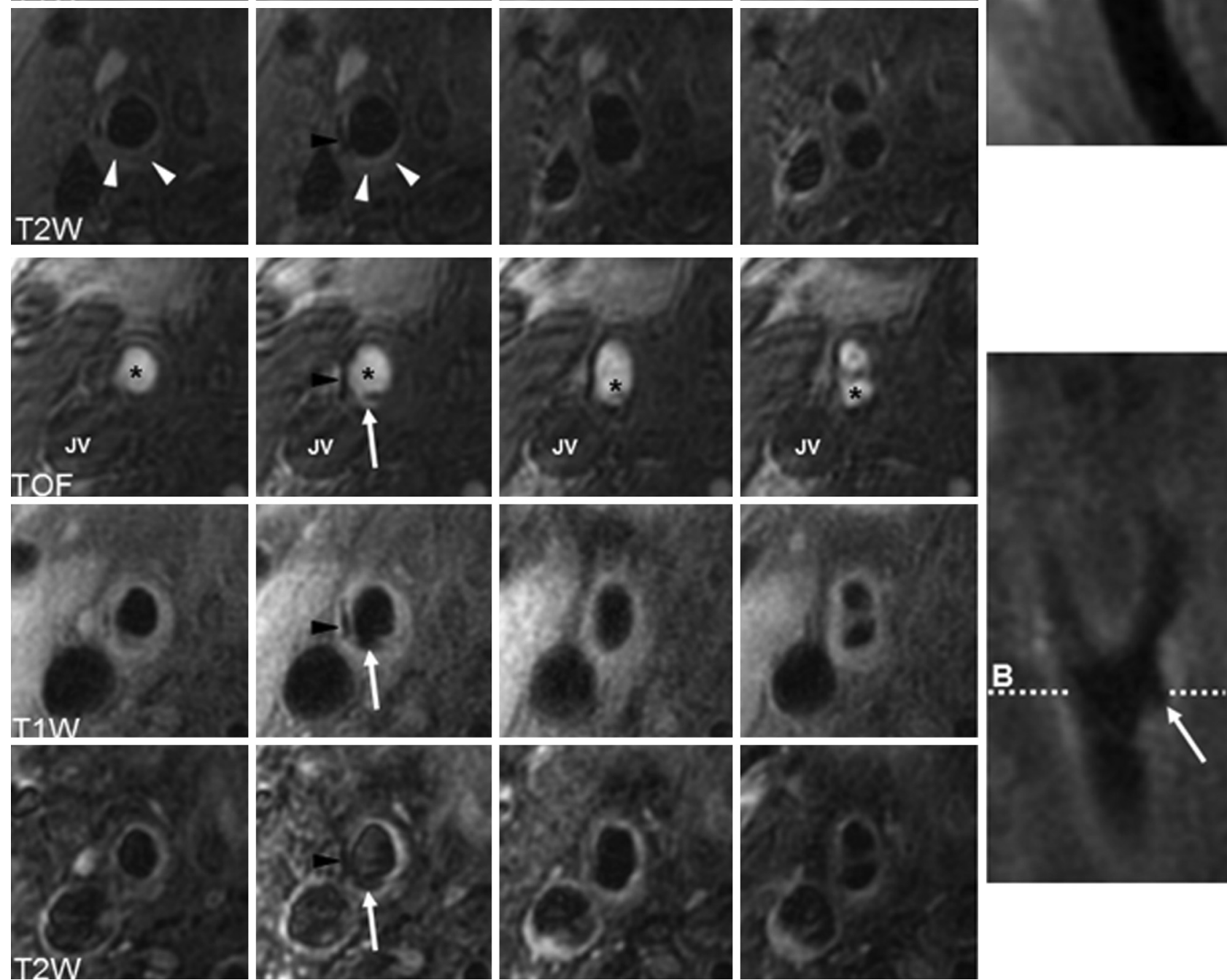

T2W
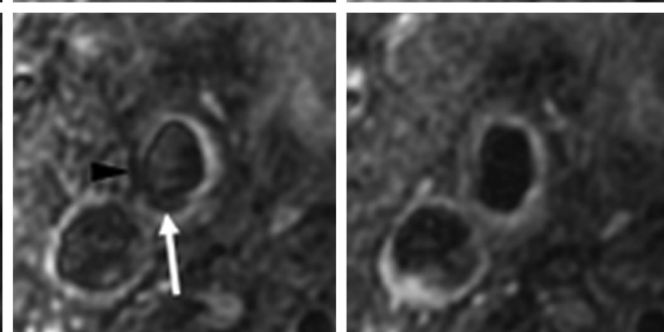

Fig 2. Multicontrast images from the right carotid artery of a 78-year-old man. Baseline images (top panel) demonstrate the presence of a relatively small lesion and minimal lumen narrowing, but a large portion of the arterial wall is occupied by the LRNC. There is an identifiable band of tissue separating the LRNC (white arrowheads, hypointense signal intensity on T2) from the lumen on T2, which is consistent with the presence of a thick fibrous cap. At 3 years (bottom panel), there is an identifiable surface disruption (white arrow) on both axial images and the longitudinal scout image. The dashed line on the longitudinal image represents the imaging location of the axial image in column $B$. IPH is not present at baseline or at follow-up, but there is visual evidence of an increase in plaque burden and a reduction in lumen area, particularly in column $D$. The asterisk indicates the lumen of either the common carotid artery or the internal carotid artery; the black arrowhead, calcification.

with the development of surface disruption during binary logistic univariate analysis are listed in Table 2. Results of ROC analysis and a comparison between lesions with and without new surface disruption for each metric are also provided (Table 2). All lesions that developed a new surface disruption had an LRNC at baseline. The percentage LRNC volume was the 


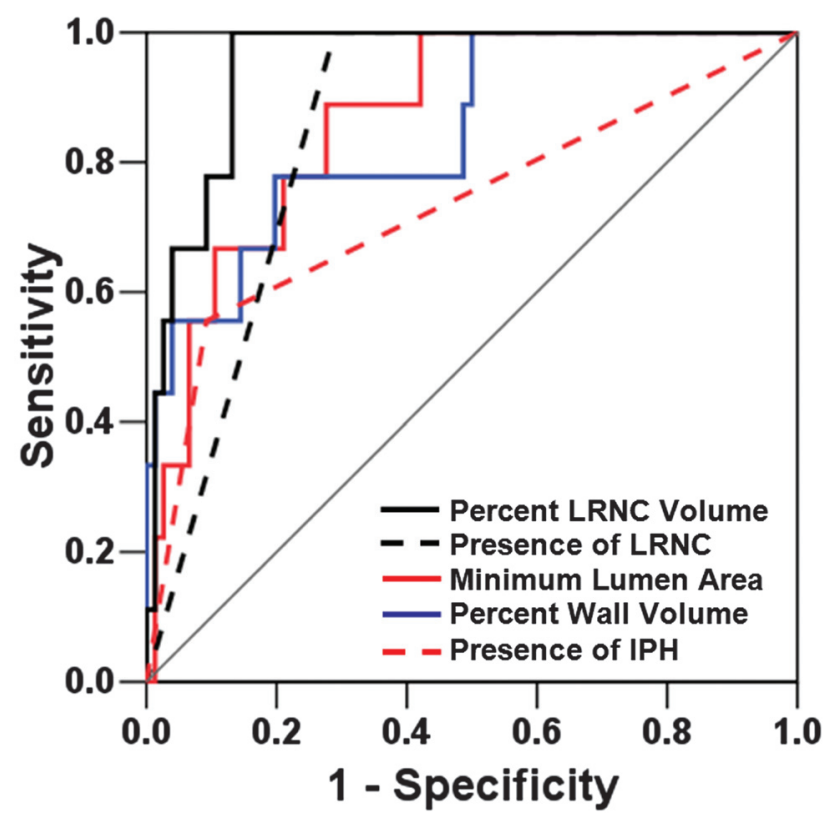

Fig 3. Plots from ROC analysis for development of a new surface disruption.

strongest classifier for predicting the development of a new surface disruption (AUC $=0.95$, Fig 3). Clinical variables (listed in Table 1) were not significantly associated with the occurrence of a surface disruption.

\section{Plaque Progression and New Surface Disruption}

Annualized rates of change in plaque morphology and composition are detailed in Table 3 for lesions that developed a new surface disruption compared with lesions that remained intact during the period of observation. In lesions with a new surface disruption, the percentage LRNC volume increased significantly faster compared with lesions with an intact surface $(1.7 \pm 2.0 \%$ versus $0.1 \pm 0.7 \%$ per year, respectively; $P=$ $.049)$.

\section{Cerebrovascular Symptoms}

During the 3-year period of observation, 3.7\% (4/108) of participants developed cerebrovascular symptoms in the vascular distribution of the index carotid artery. Two subjects developed symptoms coincident with their 3-year follow-up scan. The other 2 subjects developed symptoms before the 3-year follow-up MR imaging: 1 subject elected medical therapy and the other was not a candidate for surgery due to comorbidities. Three of the 4 (75\%) symptomatic subjects had an IPH at baseline, and 2 of these $3(67 \%)$ had a fibrous cap rupture at baseline. The fourth symptomatic individual demonstrated imaging evidence of a calcified nodule and had neither an IPH nor a fibrous cap rupture at baseline. Because the study sample was a subgroup of the individuals evaluated by Takaya et al, ${ }^{5}$ we refer the reader to this previous work for a complete analysis of the association between baseline arterial and clinical features and the development of neurologic symptoms. In the present study, none (0/9) of the individuals who developed a new fibrous cap rupture became symptomatic during the 3-year period of follow-up.

\section{Discussion}

In this prospective observational study of asymptomatic individuals with $50 \%-79 \%$ stenosis, we found a striking association between the proportion of the wall occupied by the LRNC at baseline and the development of a new surface disruption. Furthermore, development of a new surface disruption was significantly associated with an increase in the proportion of arterial wall volume occupied by the LRNC. Identification of lesions at risk of developing a new surface disruption may prove clinically valuable for preventing the transition from stable to unstable atherosclerotic disease.

Severity of stenosis has been the traditional imaging criterion for atherosclerotic risk stratification. ${ }^{25}$ In this investigation, there was a propensity for surface disruption to develop in lesions with a smaller luminal area at baseline. However, minimal luminal area was not the best classifier of surface disruption. This result is consistent with data from the Asymptomatic Carotid Atherosclerosis Study, which evaluated 1662 subjects with $>60 \%$ stenosis and found that 17 carotid endarterectomies were necessary to prevent a single stroke during 5 years. ${ }^{26}$ In addition, the European Carotid Surgery Trial reported that $43.8 \%$ of the 3018 individuals with symptomatic carotid disease had $<30 \%$ stenosis on angiography, ${ }^{27}$ and the North American Symptomatic Carotid Endarterectomy Trial reported that of the 2226 symptomatic subjects with $<70 \%$ stenosis, $61 \%$ had $<50 \%$ stenosis. ${ }^{28}$ The limits of stenosis as a predictor of plaque stability are primarily driven by the outward expansion of the arterial wall in response to an increase in plaque burden to preserve luminal dimensions. ${ }^{29}$ This adaptive arterial remodeling phenomenon has been previously attributed to the poor concordance between luminal stenosis and the severity of plaque burden. ${ }^{30}$ As such, complementary imaging criteria that evaluate the arterial wall may provide a better assessment of plaque stability.

In the coronary artery, the size of the LRNC has been associated with surface disruption. ${ }^{31}$ Analysis of carotid endarterectomy specimens have found that plaques with surface ulceration and subintimal hemorrhage contain more cholesterol and less collagen than plaques without these features. ${ }^{32}$ Evaluation of carotid lesions with sonography has previously demonstrated that the relative risk of cerebrovascular events increased monotonically with the extent of plaque echolucency,

\begin{tabular}{lccc}
\hline \multicolumn{2}{l}{ Table 3: New surface disruption and plaque progression $(\boldsymbol{n}=\mathbf{8 5})$} & & \\
\hline$\Delta /$ Year & Present $(n=9)$ & $-0.3 \pm 1.4(0.090)^{\mathrm{b}}$ & \\
\hline Minimum lumen area $\left(\mathrm{mm}^{2} / \mathrm{yr}\right)$ & $-0.6 \pm 1.1(0.175)^{\mathrm{b}}$ & $0.6 \pm 1.2(<0.001)^{\mathrm{b}}$ & .6 \\
Percentage wall volume $(\% / \mathrm{yr})$ & $1.6 \pm 2.1(0.048)^{\mathrm{b}}$ & $0.1 \pm 0.7(0.098)^{\mathrm{b}}$ & .20 \\
Percentage LRNC volume (\%/yr) & $1.7 \pm 2.0(0.035)^{\mathrm{b}}$ & $0.2 \pm 0.8(0.030)^{\mathrm{b}}$ & .049 \\
Percentage calcification volume $(\% / \mathrm{yr})$ & $0.2 \pm 1.3(0.69)^{\mathrm{b}}$ & .93 \\
\hline
\end{tabular}


a marker of the LRNC. ${ }^{33}$ Our prospective data indicated that the proportion of the arterial wall occupied by the LRNC was a better classifier of future surface disruption than several known indicators of plaque instability, including IPH and plaque burden. Collectively these findings offer compelling evidence that the metrics associated with the LRNC may provide a better index of plaque vulnerability for new surface disruption than other metrics of lesion morphology and plaque composition.

Two study limitations merit consideration. Although 108 individuals were serially evaluated and monitored during an interval of 3 years, only $3.7 \%$ of individuals had clinical events. As such, statistically significant associations were not observed between the occurrence of a new surface disruption and the development of symptoms. Of note, Takaya et $\mathrm{al}^{5}$ have previously reported a stronger association with the prospective development of neurologic symptoms for fibrous cap status and IPH at baseline compared with size of the LRNC at baseline. Accordingly, lesions with IPH and/or surface disruption may pose a greater clinical risk for development of subsequent symptoms than lesions with only a large LRNC. However, the temporal association between the development of surface disruption in lesions with a large LRNC and the subsequent occurrence of embolic events remains ambiguous. Larger long-term prospective studies are necessary to differentiate lesion severity better on the basis of the presence, absence, or combination of high-risk features (eg, IPH, surface disruption, large LRNC) at baseline. Nevertheless, the findings presented herein offer exciting evidence that plaques with a larger LRNC are more susceptible to a future surface disruption.

Second, serial brain MR imaging of all subjects was not performed. In the Cardiovascular Health Study of 3324 asymptomatic individuals, $28 \%$ of participants had evidence of silent infarcts on MR imaging at baseline. ${ }^{34}$ Additionally, there were 1433 participants who underwent 2 MR imaging scannings separated by 5 years and had no infarcts on initial MR imaging. On follow-up MR imaging, $17.7 \%$ had $\geq 1$ infarcts; and of these individuals, only $11.4 \%$ were symptomatic. ${ }^{35}$ While not all of the events in these studies ${ }^{34,35}$ were attributable to carotid disease, these findings indicate that the effects of carotid atherosclerotic disease, including surface disruption, would be better understood if future studies evaluate silent ischemia.

\section{Conclusions}

The proportion of wall volume occupied by the LRNC was the strongest classifier for predicting a new surface disruption. Furthermore, carotid plaques that developed new surface disruption demonstrated more rapid progression in percentage LRNC. These initial findings demonstrate the value of highresolution carotid MR imaging in prospective studies investigating the mechanisms of plaque progression and form the basis for larger studies on the relationship between predictors of plaque disruption, its role in the etiology of future ischemic events, and the efficacy of aggressive preventative medical therapy.

\section{References}

1. Sitzer M, Muller W, Siebler M, et al. Plaque ulceration and lumen thrombus are the main sources of cerebral microemboli in high-grade internal carotid artery stenosis. Stroke 1995;26:1231-33

2. Park AE, McCarthy WJ, Pearce WH, et al. Carotid plaque morphology correlates with presenting symptomatology. J Vasc Surg 1998;27:872-78, discussion 878-79

3. Saam T, Cai J, Ma L, et al. Comparison of symptomatic and asymptomatic atherosclerotic carotid plaque features with in vivo MR imaging. Radiology 2006;240:464-72

4. Yuan C, Zhang SX, Polissar NL, et al. Identification of fibrous cap rupture with magnetic resonance imaging is highly associated with recent transient ischemic attack or stroke. Circulation 2002;105:181-85

5. Takaya N, Yuan C, Chu B, et al. Association between carotid plaque characteristics and subsequent ischemic cerebrovascular events: a prospective assessment with MRI-initial results. Stroke 2006;37:818-23

6. Yuan C, Beach KW, Smith LH Jr, et al. Measurement of atherosclerotic carotid plaque size in vivo using high resolution magnetic resonance imaging. $\mathrm{Circu}$ lation 1998;98:2666-71

7. Hatsukami TS, Ross R, Polissar NL, et al. Visualization of fibrous cap thickness and rupture in human atherosclerotic carotid plaque in vivo with high-resolution magnetic resonance imaging. Circulation 2000;102:959-64

8. Saam T, Ferguson MS, Yarnykh VL, et al. Quantitative evaluation of carotid plaque composition by in vivo MRI. Arterioscler Thromb Vasc Biol 2005;25:234-39

9. Chu B, Kampschulte A, Ferguson MS, et al. Hemorrhage in the atherosclerotic carotid plaque: a high-resolution MRI study. Stroke 2004;35:1079-84

10. Mitsumori LM, Hatsukami TS, Ferguson MS, et al. In vivo accuracy of multisequence MR imaging for identifying unstable fibrous caps in advanced human carotid plaques. J Magn Reson Imaging 2003;17:410-20

11. Murphy RE, Moody AR, Morgan PS, et al. Prevalence of complicated carotid atheroma as detected by magnetic resonance direct thrombus imaging in patients with suspected carotid artery stenosis and previous acute cerebral ischemia. Circulation 2003;107:3053-58

12. Saam T, Yuan C, Chu B, et al. Predictors of carotid atherosclerotic plaque progression as measured by noninvasive magnetic resonance imaging. Atherosclerosis 2007;194:e34-42

13. Takaya N, Yuan C, Chu B, et al. Presence of intraplaque hemorrhage stimulates progression of carotid atherosclerotic plaques: a high-resolution magnetic resonance imaging study. Circulation 2005;111:2768-75

14. Underhill HR, Yuan C, Zhao XQ, et al. Effect of rosuvastatin therapy on carotid plaque morphology and composition in moderately hypercholesterolemic patients: a high-resolution magnetic resonance imaging trial. Am Heart J 2008; 155:584.el-8. Epub 2008 Jan 18

15. Corti R, Fuster V, Fayad ZA, et al. Lipid lowering by simvastatin induces regression of human atherosclerotic lesions: two years' follow-up by high-resolution noninvasive magnetic resonance imaging. Circulation 2002;106: 2884-87

16. Adams GJ, Greene J, Vick GW 3rd, et al. Tracking regression and progression of atherosclerosis in human carotid arteries using high-resolution magnetic resonance imaging. Magn Reson Imaging 2004;22:1249-58

17. Roederer GO, Langlois YE, Yager KA, et al. A simple spectral parameter for accurate classification of severe carotid disease. Bruit 1984;8:174-78

18. Yuan C, Kerwin WS, Yarnykh VL, et al. MRI of atherosclerosis in clinical trials. NMR Biomed 2006;19:636-54

19. Kerwin $\mathrm{W}, \mathrm{Xu} \mathrm{D}$, Liu F, et al. Magnetic resonance imaging of carotid atherosclerosis: plaque analysis. Top Magn Reson Imaging 2007;18:371-78

20. Nissen SE, Tuzcu EM, Schoenhagen P, et al. Effect of intensive compared with moderate lipid-lowering therapy on progression of coronary atherosclerosis: a randomized controlled trial. JAMA 2004;291:1071-80

21. Saam T, Hatsukami TS, Yarnykh VL, et al. Reader and platform reproducibility for quantitative assessment of carotid atherosclerotic plaque using 1.5T Siemens, Philips, and General Electric scanners. J Magn Reson Imaging 2007;26: $344-52$

22. Takaya N, Cai J, Ferguson MS, et al. Intra- and interreader reproducibility of magnetic resonance imaging for quantifying the lipid-rich necrotic core is improved with gadolinium contrast enhancement. J Magn Reson Imaging 2006;24:203-10

23. Saam T, Kerwin WS, Chu B, et al. Sample size calculation for clinical trials using magnetic resonance imaging for the quantitative assessment of carotid atherosclerosis. J Cardiovasc Magn Reson 2005;7:799-808

24. Underhill HR, Yarnykh VL, Hatsukami TS, et al. Carotid plaque morphology and composition: initial comparison between 1.5- and 3.0-T magnetic field strengths. Radiology 2008;248:550-60

25. Moore WS, Barnett HJ, Beebe HG, et al. Guidelines for carotid endarterectomy: a multidisciplinary consensus statement from the Ad Hoc Committee, American Heart Association. Circulation 1995;91:566-79

26. Endarterectomy for asymptomatic carotid artery stenosis: Executive Committee for the Asymptomatic Carotid Atherosclerosis Study. JAMA 1995;273:1421-28 
27. Rothwell PM, Gutnikov SA, Warlow CP. Reanalysis of the final results of the European Carotid Surgery Trial. Stroke 2003;34:514-23

28. Barnett HJ, Taylor DW, Eliasziw M, et al. Benefit of carotid endarterectomy in patients with symptomatic moderate or severe stenosis: North American Symptomatic Carotid Endarterectomy Trial Collaborators. N Engl J Med 1998;339:1415-25

29. Glagov S, Weisenberg E, Zarins CK, et al. Compensatory enlargement of human atherosclerotic coronary arteries. $N$ Engl J Med 1987;316:1371-75

30. Babiarz LS, Astor B, Mohamed MA, et al. Comparison of gadolinium-enhanced cardiovascular magnetic resonance angiography with high-resolution black blood cardiovascular magnetic resonance for assessing carotid artery stenosis. J Cardiovasc Magn Reson 2007;9:63-70
31. Falk E. Why do plaques rupture? Circulation 1992;86:III30-42

32. Seeger JM, Barratt E, Lawson GA, et al. The relationship between carotid plaque composition, plaque morphology, and neurologic symptoms. J Surg Res 1995;58:330-36

33. Mathiesen EB, Bonaa $\mathrm{KH}$, Joakimsen O. Echolucent plaques are associated with high risk of ischemic cerebrovascular events in carotid stenosis: the Tromso study. Circulation 2001;103:2171-75

34. Bernick C, Kuller L, Dulberg C, et al. Silent MRI infarcts and the risk of future stroke: the cardiovascular health study. Neurology 2001;57:1222-29

35. Longstreth WT Jr, Dulberg C, Manolio TA, et al. Incidence, manifestations, and predictors of brain infarcts defined by serial cranial magnetic resonance imaging in the elderly: the Cardiovascular Health Study. Stroke 2002;33:2376-82 